\title{
Patient's experiences with the care for juvenile idiopathic arthritis across Europe
}

E. H. Pieter. van Dijkhuizen ${ }^{1}$, Tsipi Egert ${ }^{2}$, Yona Egert ${ }^{2}$, Wendy Costello ${ }^{3}$, Casper Schoemaker ${ }^{1,4}$, Marlous Fernhout ${ }^{4}$, Mirjam Kepic ${ }^{5}$, Alberto Martini ${ }^{6}$, Silvia Scala ${ }^{6}$, Ingrid Rotstein-Grein ${ }^{1,7}$, Sebastiaan J. Vastert ${ }^{1}$ and Nico M. Wulffraat ${ }^{*}$

\begin{abstract}
Background: To assess the views of juvenile idiopathic arthritis (JIA) patients and their parents on the care and treatment they receive in referral pediatric rheumatology centers throughout Europe.

Methods: In a collaboration between physicians and patient associations, a questionnaire was developed, covering various domains of JIA care, including demographics, diagnosis, referrals to various health care professionals, access to pain and fatigue management and support groups, information they received about the disease and awareness of and participation in research. The questionnaire was translated and distributed by parent associations and pediatric rheumatologists in 25 countries, 22 of which were European. After completion the replies were entered on the PRINTO website. Replies could either be entered directly by parents on the website or on paper. In these cases, the replies were scanned and emailed by local hospital staff to Utrecht where they were entered by I.R. in the database.
\end{abstract}

Results: The survey was completed by 622 parents in 23 countries. The majority (66.7\%) of patients were female, with median age 10-11 years at the completion of the questionnaire. Frequencies of self-reported JIA categories corresponded to literature. Some patients had never been referred to the ophthalmologist (22.8\%) or physiotherapist (31.7\%). Low rates of referral or access to fatigue (3.5\%) or pain management teams (10.0\%), age appropriate disease education (11.3\%), special rehabilitation (13.7\%) and support groups (20.1\%) were observed. Many patients indicated they did not have contact details for urgent advice (35.9\%) and did not receive information about immunizations (43.2\%), research (55.6\%) existence of transition of care clinics (89,2\%) or financial support (89.7\%). While on immunosuppressive drugs, about one half of patients did not receive information about immunizations, travelling, possible infections or how to deal with chickenpox or shingles.

Conclusions: Low rates of referral to health care professionals may be due to children whose illness is well managed and who do not need additional support or information. Improvements are needed, especially in the areas of supportive care and information patients receive. It is also important to improve doctor patient communication between visits. Physicians can be instrumental in the setting up of support groups and increasing patients' awareness of existing support. Suggestions are given to convey crucial pieces of information structurally and repeatedly to ensure, among other things, compliance.

Keywords: Patient participation, Care, Pediatric rheumatology, Europe

\footnotetext{
* Correspondence: N.Wulffraat@umcutrecht.nl

'Paediatric Rheumatology, Wilhelmina Children's Hospital, University Medical Centre Utrecht, Room KC.03.063.0, P.O. box 85090, 3508, AB, Utrecht, The Netherlands

Full list of author information is available at the end of the article
} 


\section{Background}

There is much ongoing research in the realm of pediatric rheumatologic diseases in general, and juvenile idiopathic arthritis (JIA) specifically. The ultimate aim of this research is to provide benefits to the patients, in terms of better disease characterization, increased treatment efficacy and improved outcomes. To achieve this, a recent development has been to put more emphasis on the patient's opinion in direct interaction between health care providers and patients advocacy groups and using socalled patient reported outcomes (PROs). [1] Indeed, the patient, as the most important stakeholder is pivotal in the evaluation of disease outcome. Active involvement of patients in evaluation of care and research is now a common goal for professional and patient organizations [2-4].

Consequently, the patients' perspective with respect to their care and treatment is of utmost importance to identify areas in need of improvement. The patients' and their parents' views give insights into the organization of care for JIA patients in different countries, how the care is perceived by the patients and if it corresponds to existing guidelines and recommendations. Furthermore, we can find out if the patient has access to the range of services that exist or are needed. Additionally, investigation of the patients' perspective brings to light if information given over to the patient is understood and retained. This is an important question, since it is known that during emotional stress little of the information received is remembered [5].

Armed with knowledge about these facets, specific points can be addressed to improve JIA care and to increase the patients' awareness of available care where there is a discrepancy between the actual availability and the patients' appreciation of it. Improved communication between physicians and patients can contribute to compliance (the parent understands what needs to be done and why), and patient satisfaction [6-10].

Therefore, the aim of this study was to survey a big panel of parents of JIA patients in a large number of mainly European countries regarding their perspectives on the care and treatment they receive.

\section{Methods}

The study was performed by collaborators from the SHARE project, the Pediatric Rheumatology European Society (PReS) and the European Network for Children with Arthritis (ENCA). The survey was part of a larger project called SHARE conducted with the aim of optimizing and standardizing JIA care [11].

\section{Questionnaire description}

The questions of the survey were grouped into four broad categories: (1) demographics and diagnosis; (2) organization of care; (3) social aspects; and (4) transition to adult care. In the demographics domain, specific questions included the age of the child at the time of completion of the questionnaire, sex and JIA category. In the questions about the organization of care, respondents were asked to give an indication of the waiting time between referral and the first visit, as well as of the interval between visits and the duration of the first and subsequent visits. Referrals to other health care professionals, such as physiotherapists, ophthalmologists and orthopedic surgeons, were also surveyed. Parents were asked if they received information about care and treatment, immunizations, schools and employers, nutritional needs, ongoing research, what to do in case of disease worsening, as well as if they knew about support groups (including financial support). The questionnaire covered information received upon starting biological medications as well. Specific attention was paid to visits to the ophthalmologist and the frequency as well as the location of these visits. A group of questions was dedicated to research: Parents were asked to indicate if they were aware of registries and drug trials and if their child participated in any of these. Access to different health care services was surveyed as well, such as pain management, fatigue management and rehabilitation.

The social domain of the questionnaire covered questions pertaining to awareness of the existence of and access to support groups, as well as if the school the child was attending had been contacted by a member of the rheumatology team. Finally, the domain pertaining to transition care asked if parents and their children received information about the transitioning to adult care and addressed specific adolescent issues, such as alcohol, drugs, sexual health and pregnancies. The full questionnaire is included in the supplementary material.

The questionnaire was distributed using two main routes: Through pediatric rheumatologists who were members of the pediatric rheumatology international trials organization (PRINTO) in 25 countries, and by the individual parent associations in each country, thus possibly including JIA patients treated by physicians other than pediatric rheumatologists. The replies were not monitored for missing values or inconsistencies. The questionnaire was completed anonymously by patients with JIA according to international league against rheumatism (ILAR) criteria.

Review by the local ethics committees was not required, since this research was initiated by the patients themselves and included a questionnaire only.

\section{Statistical analysis}

The anonymized survey results were summarized by calculating the median, 1st quartile and 3rd quartile for continuous data and the number and frequency for categorical data. Countries were categorized into western 
and non-western countries (Additional file 1: Table S1) and differences between these regions were tested using Pearson's $X^{2}$-test or Fisher's exact test for categorical data and the Mann-Whitney $U$ test for continuous data.

Statistical analysis was carried out using R 3.3.2 ( $R$ Foundation for statistical computing, Vienna, Austria).

\section{Results}

\section{General demographics}

The questionnaire was completed by 622 patients in 23 countries (420 patients, $67.5 \%$, in 15 western countries and 202 patients, $32.5 \%$, in 8 non-western countries; Table 1; Additional file 1: Tables S1 and S2). No responses were obtained from centers in two countries. The majority of patients were female (66.7\%) and the median (1st quartile-3rd quartile) age at the time of completion of the questionnaire was 10 years $(7-15)$ in western countries and 11 years (8-15) in non-western countries. There were no differences in sex or age distribution between western and non-western countries (Table 1). The median age at diagnosis was equal in both western and non-western countries (5 years; Table 1 ). The most frequent self-reported JIA categories were oligoarthritis $(25.2 \%$ and $30.7 \%$ in western and nonwestern countries, respectively) and rheumatoid factor (RF) negative polyarthritis $(24.5 \%$ and $20.3 \%$ in western and non-western countries, respectively). There were differences mainly in the proportion of patients indicating undifferentiated arthritis and an unknown category of JIA between western and non-western countries (Table 1).

\section{Referrals and intervals between visits}

The median (1st quartile-3rd quartile) time between the referral and the first visit was shorter in western countries (14 [5-30] days) when compared to nonwestern countries 21 7-61] days; $p=0.001$ ). The median time interval between two visits was equal for both regions (3 months). In non-western countries, 155 respondents (76.7\%) replied that more than $45 \mathrm{~min}$ were allotted to the first visit, compared to 238 respondents $(56.7 \% ; p<0.0001)$ in western countries. Likewise, more respondents in non-western countries indicated that more than 25 min were allotted to follow up visits $(n=148,73.3 \%$, compared to $n=185,44.0 \% ; p<0.0001)$.

Ophthalmology, physiotherapy, and multidisciplinary care The majority of respondents had been referred to the ophthalmologist at least once (Table 2). Almost 4 out of 10 respondents were being screened at least every 3 months $(n=244,39.2 \%)$ and $46.3 \%(n=288)$ were being screened every 6 months. The data collected did not include information about how many of the patients had uveitis at the time of the survey. There were no differences between western and non-western countries.

More patients in non-western countries had visited the physiotherapist compared to western patients (Table 2). Most patients had never been referred to any of the following health professionals: occupational therapist, social worker, psychologist or orthodontist (Table 2). Moreover, only a minority of patients indicated having access to pain and fatigue management, support groups, age appropriate disease education and special rehabilitation. A minority in

Table 1 Patient demographics

\begin{tabular}{|c|c|c|c|}
\hline & Western countries & Non-western countries & $P$-value ${ }^{a}$ \\
\hline N & 420 & 202 & \\
\hline Female, $n(\%)$ & $286(68.1 \%)$ & $129(63.9 \%)$ & 0.34 \\
\hline Age at completion, median [1st quartile, 3rd quartile] & $10[7,15]$ & $11[8,15]$ & 0.50 \\
\hline Age at diagnosis, median [1st quartile, 3rd quartile] & $5[2,9]$ & $5[3,10]$ & 0.04 \\
\hline \multicolumn{4}{|l|}{ Self-reported JIA category, $n$ (\%) } \\
\hline Systemic & $57(13.6 \%)$ & $23(11.4 \%)$ & 0.53 \\
\hline Oligoarthritis & $106(25.2 \%)$ & $62(30.7 \%)$ & 0.18 \\
\hline Polyarthritis RF - & $103(24.5 \%)$ & $41(20.3 \%)$ & 0.29 \\
\hline Polyarthritis RF + & $46(11.0 \%)$ & $14(6.9 \%)$ & 0.15 \\
\hline Psoriatic & $21(5.0 \%)$ & $5(2.5 \%)$ & 0.21 \\
\hline ERA & $25(6.0 \%)$ & $12(5.9 \%)$ & $>0.99$ \\
\hline Undifferentiated & $19(4.5 \%)$ & $1(0.5 \%)$ & 0.02 \\
\hline Don't know & 43 (10.2\%) & $44(21.8 \%)$ & 0.0002 \\
\hline
\end{tabular}

${ }^{a}$-value of the test of differences between western and non-western countries. For categorical data Pearson's $X^{2}$-test was used; for continuous data the Mann-Whitney $\mathrm{U}$ test

Abbreviations: ERA enthesitis-related arthritis, JIA juvenile idiopathic arthritis, $R F$ rheumatoid factor 
Table 2 Referrals and access to supportive care

\begin{tabular}{|c|c|c|c|}
\hline & Western countries & Non-western countries & $P$-value \\
\hline N & 420 & 202 & \\
\hline \multicolumn{4}{|l|}{ Referrals to: } \\
\hline Ophthalmologist & $327(77.9 \%)$ & $153(75.7 \%)$ & 0.63 \\
\hline Physiotherapist & $270(64.3 \%)$ & $155(76.7 \%)$ & 0.002 \\
\hline Occupational therapist & $93(22.1 \%)$ & $8(4.0 \%)$ & $<0.0001$ \\
\hline Social worker & $44(10.5 \%)$ & $1(0.5 \%)$ & $<0.0001$ \\
\hline Psychologist & 95 (22.6\%) & $19(9.4 \%)$ & 0.0001 \\
\hline Orthodontist & 79 (18.8\%) & $60(29.7 \%)$ & 0.003 \\
\hline \multicolumn{4}{|l|}{ Indicated access to: } \\
\hline Pain management & $54(12.9 \%)$ & $8(4.0 \%)$ & 0.0009 \\
\hline Fatigue management & $16(3.8 \%)$ & $6(3.0 \%)$ & 0.77 \\
\hline Support group & $108(25.7 \%)$ & $17(8.4 \%)$ & $<0.0001$ \\
\hline Age appropriate disease education & $49(11.7 \%)$ & $21(10.4 \%)$ & 0.74 \\
\hline Special rehabilitation & $36(8.6 \%)$ & $49(24.3 \%)$ & $<0.0001$ \\
\hline Counselling and psychological support & $177(42.1 \%)$ & 35 (17.3\%) & $<0.0001$ \\
\hline
\end{tabular}

${ }^{a} P$-value of the test of differences between western and non-western countries, using Pearson's $x^{2}$-test

both regions indicated having access to counselling and psychological support (Table 2). The majority of patients from western countries $(n=348,82.9 \%)$ were aware of the existence of patients groups, forums or network meetings, compared to a minority of non-westerners $(n=45,22.3 \%$; $p<0.0001)$. Few respondents $(n=67,10,8 \%)$ indicated they were aware of the presence of a transition of care clinic in their center, This was similar is western and nonwestern countries.

\section{Emergency information and maintaining contact with doctor between visits}

The majority of patients received information about ongoing care and treatment. There was an indication that non-western patients received more often information about what to do in case of worsening symptoms (Table 3). Fewer westerners than non-westerners indicated having received contact details for urgent advice. Overall, more non-westerners than westerners could contact their pediatric center by phone and had a telephone help line or e-mail contact available. Western patients experienced longer waits for a response more frequently compared to non-western patients (Table 3).

\section{Doctors as a source of specific and general JIA} information, including information about immunizations Many patients reported that they did not get information about immunizations, support groups, financial support, school or employers, and healthy eating (>40\%). Overall, patients in western countries received more frequently information about support groups, whereas patients in nonwestern countries received more frequently information about immunizations, school or employers and healthy eating (Table 3). With respect to information received regarding immunosuppressive drugs, one third to one half of patients did not receive information about immunizations and possible infections, while more than half did not receive travel advice or guidance on how to deal with chickenpox or shingles.

Overall, non-westerners got more information than westerners (Table 3).

\section{Information about trials and research}

About one half of patients received information about research (Table 3). Slightly fewer respondents were aware that information was being collected about treatment and side effects $(n=152,36.2 \%$ in western countries and $n=104,51.5 \%$ in non-western countries; $p=0.0004)$. More western than non-western patients had been approached to enter a trial $(n=169,40.2 \%$ vs. $n=29,14.4 \% ; p<0.0001)$. Conversely, non-western patients participated more frequently in a registry than western patients $(n=75,37.1 \%$ vs. $n=93$, $22.1 \% ; p=0.0001)$.

\section{Discussion}

The data from this survey provided a unique view regarding care, opinions, experiences and information received by 622 JIA patients and their parents, living in 21 European countries, Israel and Turkey (Additional file 1: Table S1). As such this data collection is unique and relevant for an analysis of the perceived quality of care. 
Table 3 Patient-physician communication

\begin{tabular}{|c|c|c|c|}
\hline & Western countries & Non-western countries & $P$-value ${ }^{a}$ \\
\hline N & 420 & 202 & \\
\hline \multicolumn{4}{|l|}{ Received information about: } \\
\hline Ongoing care and treatment & $372(88.6 \%)$ & $187(92.6 \%)$ & 0.16 \\
\hline What to do in case of worsening symptoms & $316(75.2 \%)$ & $168(83.2 \%)$ & 0.03 \\
\hline Contact details urgent advice & $251(59.8 \%)$ & $148(73.3 \%)$ & 0.001 \\
\hline Immunizations & $220(52.4 \%)$ & $133(65.8 \%)$ & 0.002 \\
\hline Support groups & $136(32.4 \%)$ & 17 (8.4\%) & $<0.0001$ \\
\hline Financial support & $39(9.3 \%)$ & $25(12.4 \%)$ & 0.30 \\
\hline School/employers & $87(20.7 \%)$ & $84(41.6 \%)$ & $<0.0001$ \\
\hline Healthy eating & $59(14.0 \%)$ & $62(30.7 \%)$ & $<0.0001$ \\
\hline Research & $166(39.5 \%)$ & $110(54.5 \%)$ & 0.0006 \\
\hline \multicolumn{4}{|l|}{ Appropriate advice with respect to medication: } \\
\hline Immunizations/vaccines & $251(59.8 \%)$ & $150(74.3 \%)$ & 0.0006 \\
\hline Travel advice & $130(31.0 \%)$ & $106(52.5 \%)$ & $<0.0001$ \\
\hline Possible infections & $207(49.3 \%)$ & $135(66.8 \%)$ & $<0.0001$ \\
\hline How to deal with chickenpox or shingles & $96(22.9 \%)$ & $106(52.5 \%)$ & $<0.0001$ \\
\hline \multicolumn{4}{|l|}{ Means of communication: } \\
\hline Contact paediatric centre by phone & $195(46.4 \%)$ & $144(71.3 \%)$ & $<0.0001$ \\
\hline Telephone help line & $169(40.2 \%)$ & $110(54.5 \%)$ & 0.001 \\
\hline E-mail & $212(50.5 \%)$ & $147(72.8 \%)$ & $<0.0001$ \\
\hline \multicolumn{4}{|l|}{ Waiting time reply for non-urgent questions: } \\
\hline$<24 \mathrm{~h}$ & $128(30.5 \%)$ & $67(33.2 \%)$ & 0.56 \\
\hline$>24 \mathrm{~h}$ and $<48 \mathrm{~h}$ & $140(33.3 \%)$ & $62(30.7 \%)$ & 0.57 \\
\hline$>48 \mathrm{~h}$ & $69(16.4 \%)$ & $14(6.9 \%)$ & 0.002 \\
\hline
\end{tabular}

${ }^{\mathrm{a} P} \mathrm{P}$-value of the test of differences between western and non-western countries, using Pearson's $\mathrm{X}^{2}$-test

\section{Patient's knowledge on their illness}

JIA patients seemed to be well aware of their disease category. The frequencies of the categories in our sample corresponded to reports in literature,[12] with the exception of a slightly lower frequency of oligoarthritis and a slightly higher frequency of polyarticular RF positive arthritis in western countries. This may be due to a misclassification of extended oligoarticular patients as polyarticular patients. Furthermore, the frequency of undifferentiated arthritis in our sample was much lower than the frequency reported in literature. These patients might have reported their category as unknown.

\section{Ophthalmologic screening, physiotherapy and transition of care}

Frequent ophthalmologic screenings are important for JIA patients, especially for those with positive antinuclear antibodies (ANA) [13]. In our sample, more than one in five patients indicated they did not visit the ophthalmologist. When factoring in the 80 systemic JIA patients $(12.9 \%)$, who do not need regular ophthalmologic screening, approximately $10 \%$ additional patients should be receiving regular screening, but indicated they did not.

Similarly, referrals to the physiotherapist were reported by a low percentage of patients. Physiotherapy is important because children with JIA show markedly reduced levels of physical activity and energy expenditure, regardless of disease activity [14-17]. These reduced activity levels in turn have detrimental effects on functional ability, bone mass and strength, and quality of life $[17,18]$ Moreover, pre-school aged children with JIA frequently demonstrate severe to moderate delays in the achievement of milestones [19]. Functional therapies including physiotherapy may help to overcome these detrimental effects. A number of studies have shown the potential of physical therapy as well as its safety in the non-active phase of the disease [17, 20-22].

The percentage of respondents that was aware of transition of care clinics was remarkably low, both in east and west. This was also true for parents of JIA patients over 15 years of age. This indicates that transition programs seem to start well after this age, despite the recommendation to start this process early. 
Referrals to other professionals and various support systems The rate of referrals to other professionals, such as occupational therapists, social workers and psychologists was very low in both western and non-western countries. On top of that, many respondents indicated to have no access to pain and fatigue management, support groups, age appropriate disease education and psychological support. Even though this kind of support might actually be available, these answers implied that patients were not aware of their existence. Given that pain and fatigue burden the patients' daily lives considerably [16, 23] and that many JIA patients experience difficulties at school or work, [24-28] these forms of supportive care are of utmost importance and should be made available widely.

Parent associations help to educate parents about treatments and compliance. They give information that provides practical, financial and social support to children and their families. They also support families to help their child develop happily and normally. Yet, our data indicated that parent associations do not exist in all countries (Table 2), or that, even when they do exist, doctors do not refer parents to them often enough (Table 3). Physicians can be instrumental in the creation of support groups, by helping patients get in touch with each other. On the other hand, if these support systems are already available, physicians are pivotal in making patients aware of their existence.

\section{Information about ongoing care and treatment}

Much improvement is needed with respect to informing the patient: A sizable minority of $20-25 \%$ of patients did not know what to do in case of worsening symptoms.; And only $40-50 \%$ of patients had received information about immunizations. It might be that physicians provided insufficient information to patients, [29-31] or patients may not have understood the explanation given by the physician, possibly due to the use of medical terminology [6]. Moreover, many patients indicated not having a telephone help line web-portal or e-mail contact at their disposal.

It is well known that information provided by the clinician is not always remembered by patients. This contributes to a knowledge gap that stems from emotional overload that parents experience during the first visits when much of the information is given, added to the difficulty of assimilating large amounts of new and unfamiliar information under stress. Indeed, it was previously shown that information retention is in the range of only $50-90 \%$ [7, 32, 33]. The information retention problem is worse in case of emotional stress, such as after a first visit to the oncologist [5]. Conversely, more information is remembered after follow up visits in comparison with first visits [32, 33]. The information problem is important, as effective physician-patient communication may lead to better health outcomes, increased trust and higher satisfaction [6-10]. Therefore, we suggest the following improvements to the communication:

1) The physician should repeat crucial information (including booklets and websites) starting about 3 months after the initial diagnosis and continuing up to at least 1 year, making sure that the information is understood;

2) Each visit, a short summary should be available to the patient, containing at least the following information: full name of the condition from which the child suffers, including category and ANA status; general state of health found during the visit; list of medications and dosages the child should be taking; list of laboratory and other tests that need to be performed (including tuberculosis screening when necessary). The information should include reminders for visits to the ophthalmologist and any other health care professional and a phone number or an e-mail address through which the doctor can be contacted in case of need. Early experience with providing such information by unrestricted access to medical files through a web portal indicates that only few patients wish to use this regularly;

3) Online access to medical files using portal should be encouraged. Medical files should contain a section where the goal of therapies as phrased by patients is documented.

These suggestions are in line with recent research, showing that the ability to recall information is improved by using simple language, [34] written information, [35] and the introduction of new information at intervals, when it becomes more relevant [36].

\section{Differences between western and non-western countries}

Overall, patients in western countries had access to supportive care more frequently, such as occupational therapists, social workers, psychologists, pain management and support groups. On the other hand, patients in nonwestern countries more frequently indicated referrals to the physiotherapist and the orthodontist as well as access to special rehabilitation. In between contact with centers was more frequently available for eastern country residents and they reported shorter response times, compared to western patients. Furthermore, nonwestern patients indicated having received more advice with respect to medication.

\section{Research}

We examined the patients' awareness of research, since participation in trials places them as a partner in the quest for better treatment of JIA and encourages them 
to share their experiences with treatment. Moreover, trials and registrations form an integral part of the health care in many third line centers. However, the results indicated that not many patients were aware of on-going research, let alone involved in the planning and performing of trials.

\section{Limitations}

When interpreting these data, one should take into account that the questionnaire was distributed primarily among pediatric rheumatologists, who are members of the networks of PRES, PRINTO and patient organizations. Therefore, the respondents form a biased group of JIA patients who were likely to receive the best possible care per country. Consequently, the actual situation might be less favorable than the responses indicated. This may be the case especially in non-western countries, where many JIA patients may be treated by physicians other than pediatric rheumatologists. Furthermore, the data presented in this study exclusively represented the respondents' view. The answers were not verified, for example to check whether support groups of transition of care clinics truly did not exist in case respondents indicated they did not have access to them. Therefore, in our analysis we focused on patients' awareness of supportive care, rather than its existence, as the effectiveness of supportive care is diminished when patients are unaware of its existence. Moreover, even though the questionnaire was anonymous, many physician's handed the questionnaire to the patients and received the completed form from them. This might have influenced the answers. Finally, given the big number of hypotheses tested in this manuscript, $p$-values in the range from 0.01 to 0.05 should be interpreted as providing only weak evidence [37].

\section{Conclusion}

In conclusion, the data provided a unique view on JIA patients' and their parents' perspectives regarding the care they receive. Improvements are needed mainly in the areas of supportive care and the accessibility of information to patients. The rate of referral to ophthalmologists and physiotherapists could be increased too. Suggestions have been made to convey crucial information structurally and repeatedly and to increase the awareness of the existence of supportive care, such as pain and fatigue management as well as support groups, or to start new initiatives where these are lacking.

We conclude that a sizable knowledge gap still exists in many aspects of care. Engagement of patient groups is important and a step towards active patient participation in evaluating received care and setting joint research agendas to improve this.

\section{Additional file}

Additional file 1: Table S1. Number of questionnaires per country and division into regions. Table S2. SHARE PARENT SURVEY: Patient Personal Information and Diagnosis. (DOCX 24 kb)

\begin{abstract}
Abbreviations
ANA: antinuclear antibodies (ANA; ENCA: European Network for Children with Arthritis; JIA: Juvenile Idiopathic Arthritis; PRES: Pediatric Rheumatology European Society; PRINTO: Pediatric Rheumatology International Trials Organisation; PRO: Patient Reported Outcomes; SHARE: Single Hub and Access point for pediatric Rheumatology Europe
\end{abstract}

\section{Acknowledgements}

We would like to thank all parents and patients for taking the time to fill the questionnaire.

\section{Funding}

This project was funded by a grant from European Agency for Health and Consumers (EAHC, grantnumber 2011 1202). ENCA receives financial support from the Pediatric Rheumatology European Society (PRES).

\section{Availability of data and materials}

Full data of the survey responses of individual countries and centers are available.

\section{Authors' contributions}

NW, SV and WC designed the survey. PD and TE analyzed the data and wrote the manuscript. NW and YE revised the manuscript. YE, MK, CS and WC explored the patients items. IRG designed the Tables. SS and AM provided the database and support at the PRINTO office. All authors revised and approved the manuscript.

\section{Authors' information}

Not applicable.

Ethics approval and consent to participate Not applicable.

\section{Consent for publication}

All authors have given their consent for publication.

Competing interests

The authors declare that they have no competing interests.

\section{Publisher's Note}

Springer Nature remains neutral with regard to jurisdictional claims in published maps and institutional affiliations.

\section{Author details}

'Paediatric Rheumatology, Wilhelmina Children's Hospital, University Medical Centre Utrecht, Room KC.03.063.0, P.O. box 85090, 3508, AB, Utrecht, The Netherlands. ${ }^{2}$ Inbar Parent Association, Jerusalem, Israel. ${ }^{3}$ iCAN Irish Children's Arthritis Network, Dublin, Republic of Ireland. ${ }^{4}$ Netherlands JIA patient/parent organization, Amsterdam, Netherlands. ${ }^{5}$ Slovenian JIA patient organization, Ljubljana, Slovenia. ${ }^{6}$ Paediatric Rheumatology, IRCCS G. Gaslini, Genoa, Italy. ${ }^{7}$ Department of Pediatric Rheumatology, Hospital Pequeno Príncipe, Curitiba, Paraná, Brazil.

Received: 22 November 2017 Accepted: 4 February 2018

Published online: 08 February 2018

\section{References}

1. Consolaro A, Giancane G, Schiappapietra B, Davi S, Calandra S, Lanni S, et al. Clinical outcome measures in juvenile idiopathic arthritis. Pediatr Rheumatol Online J. 2016;14(1):23.

2. Puyraimond-Zemmour D, Etcheto A, Fautrel B, Balanescu A, De WM HT, et al. Associations Between Five Important Domains of Health and the Patient Acceptable Symptom State in Rheumatoid Arthritis and Psoriatic Arthritis: A 
Cross-Sectional Study of 977 Patients. Arthritis Care Res (Hoboken ). 2017; 69(10):1504-9.

3. International Consortium for Health Outcomes Measurement (ICHOM). 2017. Ref Type: Internet Communication.

4. de Wit MP, Kvien TK, Gossec L. Patient participation as an integral part of patient-reported outcomes development ensures the representation of the patient voice: a case study from the field of rheumatology. RMD Open. 2015;1(1):e000129.

5. Dunn SM, Butow PN, Tattersall MH, Jones QJ, Sheldon JS, Taylor JJ, et al. General information tapes inhibit recall of the cancer consultation. J Clin Oncol. 1993;11(11):2279-85.

6. Korsch BM, Gozzi EK, Francis V. Gaps in doctor-patient communication. 1. Doctor-patient interaction and patient satisfaction. Pediatrics. 1968;42(5):855-71.

7. Bertakis KD. The communication of information from physician to patient: a method for increasing patient retention and satisfaction. J Fam Pract. 1977;5(2):217-22.

8. Ambady N, Koo J, Rosenthal R, Winograd CH. Physical therapists' nonverbal communication predicts geriatric patients' health outcomes. Psychol Aging. 2002;17(3):443-52.

9. Beck RS, Daughtridge R, Sloane PD. Physician-patient Communication in the primary care office: a systematic review. J Am Board Fam Pract. 2002;15(1):25-38.

10. Fiscella K, Meldrum S, Franks P, Shields CG, Duberstein P, McDaniel SH, et al. Patient trust: is it related to patient-centered behavior of primary care physicians? Med Care. 2004;42(11):1049-55.

11. Wulffraat NM, Vastert B. Time to share. Pediatr Rheumatol Online J. 2013;11(1):5.

12. Ravelli A, Martini A. Juvenile idiopathic arthritis. Lancet. 2007;369(9563):767-78.

13. Calandra S, Gallo MC, Consolaro A, Pistorio A, Lattanzi B, Bovis F, et al. Female sex and oligoarthritis category are not risk factors for uveitis in Italian children with juvenile idiopathic arthritis. J Rheumatol. 2014;41(7):1416-25.

14. Lelieveld OT, Armbrust W, van Leeuwen MA, Duppen N, Geertzen JH, Sauer PJ, et al. Physical activity in adolescents with juvenile idiopathic arthritis. Arthritis Rheum. 2008;59(10):1379-84.

15. Bos GJ, Lelieveld OT, Armbrust W, Sauer PJ, Geertzen JH, Dijkstra PU. Physical activity in children with juvenile idiopathic arthritis compared to controls. Pediatr Rheumatol Online J. 2016;14(1):42.

16. Armbrust W, Lelieveld OH, Tuinstra J, Wulffraat NM, Bos GJ, Cappon J, et al. Fatigue in patients with juvenile idiopathic arthritis: relationship to perceived health, physical health, self-efficacy, and participation Pediatr Rheumatol Online J. 2016;14(1):65.

17. Long AR, Rouster-Stevens KA. The role of exercise therapy in the management of juvenile idiopathic arthritis. Curr Opin Rheumatol. 2010;22(2):213-7.

18. Exercise KSE. In pediatric rheumatic diseases. Curr Opin Rheumatol. 2008;20(5):619-24.

19. van der Net J, van der Torre P, Engelbert RH, Engelen V, Van ZF, Takken T, et al. Motor performance and functional ability in preschool- and early school-aged children with juvenile idiopathic arthritis: a cross-sectional study. Pediatr Rheumatol Online J. 2008;6:2.

20. Catania H, Fortini V, Cimaz R. Physical exercise and physical activity for children and adolescents with juvenile idiopathic arthritis: a literature review. Pediatr Phys Ther. 2017;29(3):256-60

21. Gualano B, Bonfa E, Pereira RMR, Silva CA. Physical activity for paediatric rheumatic diseases: standing up against old paradigms. Nat Rev Rheumatol. 2017;13(6):368-79.

22. Armbrust W, Bos GJFJ, Wulffraat NM, Van BM CJ, Dijkstra PU, et al. Internet program for physical activity and exercise capacity in children with juvenile idiopathic arthritis: a multicenter randomized controlled trial. Arthritis Care Res (Hoboken ). 2017;69(7):1040-9.

23. Allen JM, Graef DM, Ehrentraut JH, Tynes BL, Crabtree VM. Sleep and pain in pediatric illness: a conceptual review. CNS Neurosci Ther. 2016;22(11):880-93.

24. Packham JC, Hall MA, Pimm TJ. Long-term follow-up of 246 adults with juvenile idiopathic arthritis: predictive factors for mood and pain. Rheumatology (Oxford). 2002;41(12):1444-9.

25. Packham JC, Hall MA. Long-term follow-up of 246 adults with juvenile idiopathic arthritis: social function, relationships and sexual activity. Rheumatology (Oxford). 2002;41(12):1440-3.
26. Packham JC, Hall MA. Long-term follow-up of 246 adults with juvenile idiopathic arthritis: education and employment. Rheumatology (Oxford). 2002;41(12):1436-9.

27. Packham JC, Hall MA. Long-term follow-up of 246 adults with juvenile idiopathic arthritis: functional outcome. Rheumatology (Oxford). 2002:41(12):1428-35.

28. Schlichtiger J, Haas JP, Barth S, Bisdorff B, Hager L, Michels H, et al. Education and employment in patients with juvenile idiopathic arthritis - a standardized comparison to the German general population. Pediatr Rheumatol Online J. 2017;15(1):45.

29. Waitzkin H. Doctor-patient Communication. Clinical implications of social scientific research. JAMA. 1984;252(17):2441-6.

30. Makoul G, Arntson P, Schofield T. Health promotion in primary care: physician-patient communication and decision making about prescription medications. Soc Sci Med. 1995;41(9):1241-54.

31. Boreham P, Gibson D. The informative process in private medical consultations: a preliminary investigation. Soc Sci Med. 1978;12(5A):409-16.

32. Ley P. Communication with patients: improving satisfaction and compliance. London: Croom Helm; 1988.

33. Hulka BS. Patient-clinician interaction. In: Haynes RB, Taylor DW, Sackett DL, editors. Compliance in health care. Baltimore: Johns Hopkins University Press; 1979.

34. Ballo P, Milli M, Slater C, Bandini F, Trentanove F, Comper G et al. Prospective validation of the Decalogue, a set of doctor-patient communication recommendations to improve patient illness experience and mood states within a hospital cardiologic ambulatory setting. Biomed Res Int 2017; 2017:2792131.

35. Kessels RP. Patients' memory for medical information. J R Soc Med. 2003;96(5):219-22.

36. Kang SHK. Spaced repetition promotes efficient and effective learning: policy implications for instruction. Policy Insights Behav Brain Sci. 2016:3(1):12-9.

37. Sterne JA, Davey SG. Sifting the evidence-what's wrong with significance tests? BMJ. 2001;322(7280):226-31.

\section{Submit your next manuscript to BioMed Central and we will help you at every step:}

- We accept pre-submission inquiries

- Our selector tool helps you to find the most relevant journal

- We provide round the clock customer support

- Convenient online submission

- Thorough peer review

- Inclusion in PubMed and all major indexing services

- Maximum visibility for your research

Submit your manuscript at www.biomedcentral.com/submit
Biomed Central 